\title{
Ultrasonography and histology correlation in BI-RADS 4/5 small breast lesions among Thai patients
}

\author{
Pimlada Chiasawas ${ }^{\mathrm{a}}$, Darunee Boonjunwetwat ${ }^{\mathrm{a}}$, Pichet Sampatanukul ${ }^{\mathrm{b}}$ \\ a'Department of Radiology; ${ }^{b}$ Department of Pathology, Faculty of Medicine, Chulalongkorn University, \\ Bangkok 10330, Thailand
}

\begin{abstract}
Background: Ultrasonography is an important imaging tool in detection of small breast cancers, particularly in younger women with dense breasts. Among the ultrasonographic characteristics for the malignancy, it is unclear which are common or more predictive.

Objective: Analyze breast ultrasonograms and determine the common and predictive characteristics of the BIRADS 4/5 small breast lesions that were correlated with histology-proved carcinoma among Thai patients.

Methods: Data were collected retrospectively between November 2006 and September 2007 at King Chulalongkorn Memorial Hospital. Forty-five BI-RADS 4 or 5 small breast lesions from 41 patients were reviewed for ultrasonographic characteristics and for correlation between each of these features and histology-proved malignancy.

Results: There were 15 out of 30 lesions of BI-RADS 4 and 14 out of 15 of BI-RADS 5 that were histologically proven breast carcinoma. The lesion dimension ranged from $0.27 \mathrm{~cm}$ to $1.5 \mathrm{~cm}$ (mean: $0.98 \mathrm{~cm}$ ). The malignant signs that were common consisted of irregular shapes $(70 \%)$ and posterior shadowing $(35.6 \%)$. However, the most correlating signs for malignancy were vascularity of the lesion $100 \%$, and spiculated margins $100 \%$. The other characteristics for malignancy, in descending order, were marked hypoechoicity $88.9 \%$, microcalcifications within mass $85.7 \%$, echogenic halo $83.3 \%$, shadowing $81.3 \%$, branched pattern $77.8 \%$, duct extension $75 \%$, irregular shape $72.2 \%$, and taller than wide orientation $70 \%$.

Conclusion: Irregular shape and shadowing were the two most common malignant signs that characterized BIRADS 4, 5 small breast lesions by ultrasonography. However, the most predictive signs were increases in vascularity and spiculated margins.
\end{abstract}

Keywords: BI-RADS 4/5, small breast cancers, ultrasonographic feature

Breast cancer is the most common cancer in females, and the age-standardized incidence rate is 37.4 per $1,000,000$ women. In Thailand, it is a leading cause of death and the second most common cancer in women. The estimated incidence rate is 20.5 per 100,000 women [1]. Early detection of small lesions might improve survival rate of patients.

Ultrasonography is most useful for detection of small breast cancers, particularly in younger women with dense breasts that are not suitable for mammography [2]. In Japan, ultrasonography could detect $15 \%$ of mammographically occult breast

Correspondence to: Darunee Boonjunwetwat MD, Department of Radiology, Faculty of Medicine, Chulalongkorn University, Bangkok 10330, Thailand. E-mail: vopun@hotmail.com cancers [3]. The proportion of ultrasonographically detected cancers among the total number of nonpalpable cancers was $22 \%$ [4]. Overall accuracy in detection of breast malignancy by ultrasonography was approximately $70 \%$. The most common mammographic parenchymal pattern of Thai women is dense breasts [5]. Therefore, it is an urgent task to establish ultrasonography for detection of small breast cancer in Thai women.

Ultrasonographic appearances can be useful in distinguishing benign and malignant lesions [6, 10-12]. Ultrasonographic characteristics of breast cancer that have been related to malignancy have been described as spiculation, taller than wide shape, angular margins, shadowing, branch pattern, hypoechogenicity, punctate calcifications, duct extension, and microlobulation [6]. 
Previous reports supported the significance of such malignant ultrasonogarphic features for small lesions $[4,6]$. However, among the ultrasonographic characteristics for the malignancy, it is still unclear which are common or predictive.

In this study, we analyzed ultrasonographic features of BI-RADS 4/5 small breast lesions to determine which are common or predictive of malignancy among Thai patients.

\section{Materials and methods}

Cases were collected retrospectively between November 2006 and September 30, 2007 at King Chulalongkorn Memorial Hospital (KCMH). This study was approved by the Ethics Committee of the Faculty of Medicine, Chulalongkorn University.

Inclusion criteria were all cases of ultrasonographic BI-RADS 4 or 5 that met the operational definition of small breast lesions and that had a subsequent histologic diagnosis.

Ultrasonographic BI-RADS 4 is a suspicious abnormality that warrants biopsy but is not diagnostic for malignancy. Lesions in this category would have an intermediate probability for cancer, ranging from $3-94 \%$. Included in this group are sonographic findings of a solid mass without all of the criteria for a fibroadenoma and of other benign lesions.
Ultrasonographic BI-RADS 5 is highly suggestive of malignancy. An abnormality identified sonographically and placed in this category should have a $95 \%$ or higher risk of malignancy so that biopsy and definitive treatment has to be considered at the outset [7].

Small breast lesions were defined as clinically nonpalpable or vague lumps where the largest dimension of the nodule by ultrasonography was not more than $1.5 \mathrm{~cm}$. Breast ultrasonography was performed with high frequency (10-12 MHz) linear transducers (Philip HDI 5000 and IU 22, Finland, GE voluson 730 expert, USA) by one experienced breast radiologist.

Ten characteristics (shape, orientation, margin, internal echogenicity, boundary echogenicity, posterior acoustic features, calcification, vascularity, branch pattern, and duct extension) were used to describe all studied nodules $[6,7]$. The number of these characteristics were tabulated and analyzed according to histology.

\section{Results}

Forty-five lesions from 41 women were diagnosed as ultrasonographic BI-RADS 4, 5 small breast lesions and had excisional biopsy results. There were multiple lesions in three patients. Table 1 shows BI-RADS 4, 5 and histologic diagnostic results.

Table 1. BI-RADS 4, 5 and histologic diagnostic results.

\begin{tabular}{llll}
\hline Histologic results & BI-RADS 4 & BI-RADS 5 & BI-RADS 4\&5 \\
\hline Malignant and benign & $\mathbf{3 0}(\mathbf{6 6 . 7 \%})$ & $\mathbf{1 5}(\mathbf{3 3 . 3} \%)$ & $\mathbf{4 5}$ \\
\hline Malignant & $\mathbf{1 5}(\mathbf{3 3 . 3 \% )}$ & $\mathbf{1 4}(\mathbf{3 1 . 1} \%)$ & $\mathbf{2 9}(\mathbf{6 4 . 4 \% )}$ \\
- invasive ductal carcinoma & $11(24.4 \%)$ & $11(24.4 \%)$ & $22(48.9 \%)$ \\
- invasive lobular carcinoma & $1(2.2 \%)$ & $2(4.4 \%)$ & $3(6.7 \%)$ \\
- ductal carcinoma in situ & $2(4.4 \%)$ & $1(2.2 \%)$ & $3(6.7 \%)$ \\
- Invasive lobular carcinoma with comedo & $1(2.2 \%)$ & 0 & $1(2.2 \%)$ \\
ductal carcinoma in situ & & & \\
Benign & $\mathbf{1 5}(\mathbf{3 3 . 3 \% )}$ & $\mathbf{1}(\mathbf{2 . 2 \% )}$ & $\mathbf{1 6}(\mathbf{3 5 . 6 \% )}$ \\
- fibrocystic change & $2(4.4 \%)$ & $1(2.2 \%)$ & $3(6.7 \%)$ \\
- fibroadenoma & $3(6.7 \%)$ & 0 & $3(6.7 \%)$ \\
- fibroadenoma with intraductal papilloma & $2(4.4 \%)$ & 0 & $2(4.4 \%)$ \\
- fibroadenoma with adenosis & $1(2.2 \%)$ & 0 & $1(2.2 \%)$ \\
- adenosis with flat epithelial atypia & $1(2.2 \%)$ & 0 & $1(2.2 \%)$ \\
- intraductal papilloma & $1(2.2 \%)$ & 0 & $1(2.2 \%)$ \\
- intraductal papilloma with atypical ductal & $1(2.2 \%)$ & 0 & $1(2.2 \%)$ \\
$\quad$ hyperplasia & $1(2.2 \%)$ & 0 & $1(2.2 \%)$ \\
- fibrocystic change with sclerosing & & & $1(2.2 \%)$ \\
$\quad$ adenosis & $1(2.2 \%)$ & 0 & $1(2.2 \%)$ \\
- fibrocystic change with fibroadenomatoid & & \\
hyperplasia & $1(2.2 \%)$ & 0 & \\
- radial scar & $1(2.2 \%)$ & 0 & \\
- unremarkable breast tissue & & & \\
\hline
\end{tabular}


Ultrasonographic characteristics of the lesions were tabulated in Table 2.
Figure 1 and 2 show several malignant ultrasonographic features of small breast lesions that had malignant histologic diagnostic results.

Table 2. The correlation between ultrasonographic features of BI-RADS 4, 5 small breast lesions and malignant histologic diagnostic results.

\begin{tabular}{lc}
\hline Characteristics & Malignant lesions/total lesions \\
\hline Vascularity in lesion & $6 / 6(100 \%)$ \\
Spiculated margin & $2 / 2(100 \%)$ \\
Markedly hypoechoic & $8 / 9(88.9 \%)$ \\
Microcalcifications within mass & $6 / 7(85.7 \%)$ \\
Echogenic halo & $5 / 6(83.3 \%)$ \\
Shadowing & $13 / 16(81.3 \%)$ \\
Branch pattern & $7 / 9(77.8 \%)$ \\
Duct extension & $9 / 12(75 \%)$ \\
Irregular shape & $26 / 36(72.2 \%)$ \\
Taller than wide orientation & $7 / 10(70 \%)$ \\
\hline
\end{tabular}
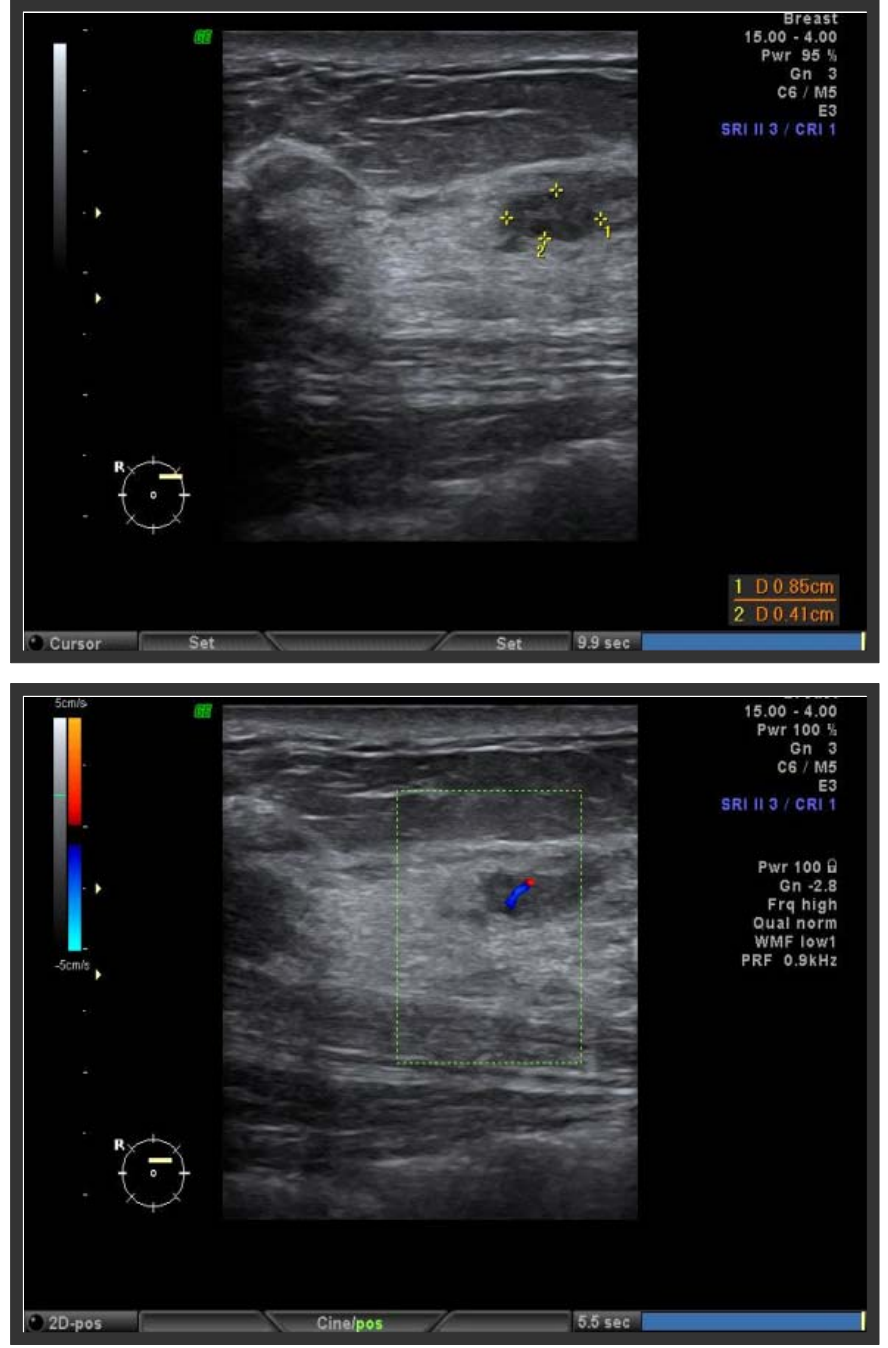

Figure 1. Ultrasonographic images showing three malignant characteristics. The lesion has irregular shape, duct extension, and vascularity in lesion. Biopsy result revealed invasive ductal carcinoma. 

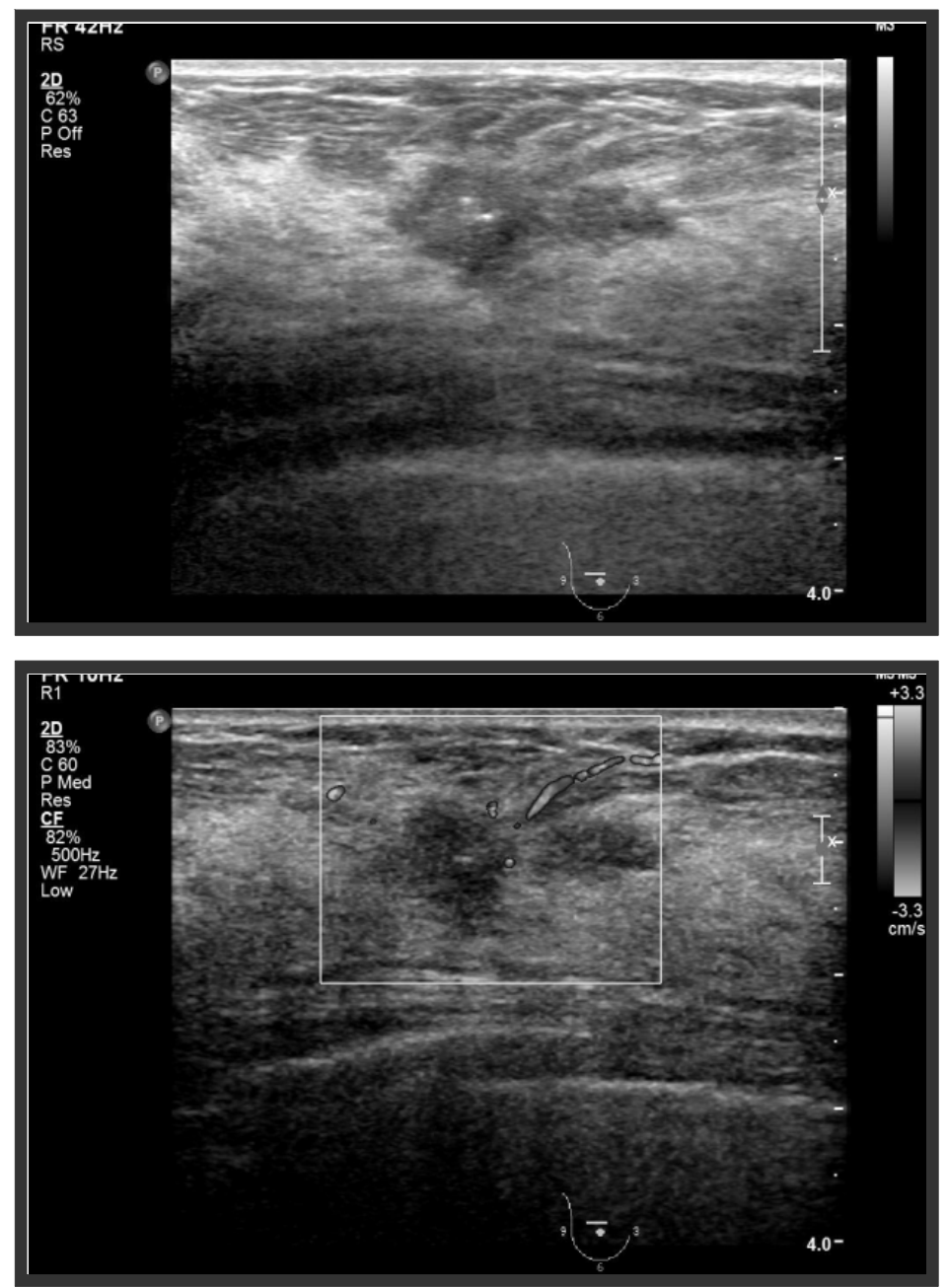

Figure 2. Ultrasonographic images showing four malignant characteristics. The lesion has irregular shape, duct extension, microcalcification within lesion and vascularity in lesion. Biopsy result revealed invasive ductal carcinoma.

\section{Discussion}

There are some controversies regarding the utility of ultrasonography when evaluating solid breast masses for the likelihood of malignancy [8,9]. However, several studies have suggested that sonographic appearances are most useful for differentiating benign from malignant solid breast masses [6, 10-12]. This study has assessed the ultrasonographic features with histology correlation in small breast cancer to identify reliable malignant features of help in the early detection of breast cancer.

In our study, the characteristic ultrasonographic features (vascularity in the lesion, spiculated margin, markedly hypoechoic, microcalcifications within mass, echogenic halo, posterior shadowing, branch pattern, duct extension, irregular shape, and taller than wide orientation) were the important imaging clues to establish the diagnosis of early breast cancer, matching other reports $[6,10,12]$. Among the characteristics, the vascularity in lesion and spiculated margin were the most significant features for diagnosis of small breast cancer as correlated with the histology. Although the growth of tumor depends on vascularization, some studies have demonstrated that the detection of color Doppler signals showed no correlation with the size of the tumor [13]. This suggests that early formation of abundant microvasculature in small tumors might be important factors. Therefore, detection of the vascularity is significant for confirmation of small breast cancer. It must be noted that vascularity cannot be detected in all malignant lesions, and the detectability of tumor vascularity depends on the ultrasound equipment. On the other hand, spiculation is an imaging feature of 
infiltrating tentacles of tumor that extend into the surrounding tissues [6]. This feature correlates well with small breast cancer in our study. In addition, the spiculation within the fibrous tissue surrounding a cancer, especially small breast lesion, may be inconspicuous mammographically and ultrasonography can be very helpful in such cases.

The imaging features of markedly hypoechoic, micro-calcifications within the mass, echogenic halo, shadowing, branch pattern, duct extension, irregular shape and taller than wide orientation could be present in both benign and malignant lesions. However, they are much more common features in malignant lesions and should be considered as malignant characteristics [6]. We have found that $88.9 \%$ of small breast cancers are markedly hypoechoic compared to fat. Therefore, a markedly hypoechoic feature is a worrisome finding for small breast cancer. Sensitivity of ultrasonography for demonstration of microcalcifications is less than with mammography [14-16]. However, the currently used high-frequency transducers can show a higher percentage of visible calcifications than the previously used lower-frequency transducers [14-16]. We have found that the majority of small breast cancer is markedly hypoechoic, and enhances our ability to identify the bright calcification echoes. Conversely, benign calcifications within a background of normal breast tissue, including large amount of hyperechoic and heterogeneous fibrous tissue, are difficult to detect sonographically [19]. Therefore, even though the sensitivity of ultrasonography for calcifications is very low compared with mammography, calcifications that are seen sonographically within a solid mass are more likely to be malignant [6]. The echogenic halo was strongly predictive of malignancy in some studies [17, 18]. However, the identification of the echogenic halo can be difficult if the tumor nidus is surrounded by hyperechoic fibroglandular tissue. The shadowing is an ultrasonographic feature of malignant nodules that has been frequently discussed in many reports papers [19-24]. In this study, shadowing was seen in a high percentage of small breast cancers (81.3\%). Variable presentation of shadowing in malignant lesions might depend on the tumor grading and type $[6,11,22,24]$. Our finding of either duct extension or a branch pattern suggests that a process might be spreading along the ductal system, increasing the likelihood of malignancy [6]. However, such extension may indicate the presence of an invasive tumor around the duct as well as an intraductal tumor [6]. An irregular shape has been a frequently reported finding and one of the most reliable findings for malignancy $[11,23,26]$. An irregular shape can indicate inconsistent growth and advancement of the lesion edge [25]. It has been reported that nodules that are taller than wider are likely to be malignant $[18,24]$. Taller-than-wider orientation on ultrasonography can suggest spread of the lesion through tissue-plane boundaries [25]. In our study, $70 \%$ of malignant lesions showed taller-than-wider lesions. Therefore, this worrisome ultrasonographic feature is more likely to be associated with small breast cancer.

In our study, the evaluation of the cases was retrospective and findings were interpreted from the static images leading to description that is more difficult to do. Therefore, although reporters were blinded to biopsy results, the observers were aware that their descriptions and assessments in BI-RADS 4/5 lesions may have been biased effecting their characterization of the lesions.

In conclusion, ultrasonography was most useful in the characterization of small breast lesion and could be helpful in evaluation of small breast lesions for malignancy.

\section{Acknowledgements}

We would like to acknowledge the assistance of Mrs. Weeranuch Kitsukjit and the Medical Records and Statistical Department of KCMH for providing the statistical data in this study. This study was performed in partial requirement for the degree of Higher Graduate Diploma of Clinical Sciences for Dr. Pimlada Chiasawas.

The authors have no conflict of interest to declare.

\section{References}

1. Chaiwerawattana A. Breast. In: Khuhaprema T, Srivatanakul P, Sriplung H, Wiangnon S, Sumitsawan Y, Attasara P, editors. Cancer in Thailand Vol.4. Bangkok: National Cancer Institute; 2007; p. 48-50.

2. Leconte I, Feger C, Galant C, Berli re M, Berg BV, D'Hoore W, et al. Mammography and subsequent whole-breast sonography of nonpalpable breast cancers: the importance of radiologic breast density. Am J Roentgenol. 2003; 180:1675-9.

3. Uchida K, Yamashita A, Kawase K, Kamiya K. Screening ultrasonography revealed $15 \%$ of mammographically occult breast cancers. Breast Cancer. 2008; 15:165-8.

4. Buchberger W, NiehoffA, Obrist P, DeKoekkoek-Doll 
P, D nser M. Clinically and mammographically occult breast lesions: detection and classification with highresolution sonography. Semin Ultrasound CT MR. 2000; 21:325-36.

5. Boonjunwetwat D, Thakhulee A, Prueksadee J. Mammographic parenchymal patterns of 1,666 Thai women: A mammographic screening evaluation. Chula Med J. 2010; 54: 303-12.

6. Stavros AT, Thickman D, Rapp CL, Dennis MA, Parker SH, Sisney GA. Solid breast nodules: use of sonography to distinguish between benign and malignant lesions. Radiology. 1995; 196:123-34.

7. American College of Radiology. ACR BI-RADSUltrasound Lexicon [online]. 2003 [cited 2008 Feb 25]. Available from: http://www.acr.org/SecondaryMain MenuCategories/quality_safety/BIRADSAtlas/BIR ADSAtlasexcerptedtext/BIRADSUltrasoundFirst Edition/ACRBIRADSUSLexiconClassificationForm Doc1.aspx.

8. Jackson VP. Management of solid breast nodules: what is the role of sonography? Radiology. 1995; 196:14-5.

9. Hall FM. Sonography of the breast: controversies and opinions. Am J Roentgenol. 1997; 169:1635-6.

10. Rahbar G, Sie AC, Hansen GC, Prince JS, Melany ML, Reynolds HE, et al. Benign versus malignant solid breast masses: US differentiation. Radiology. 1999; 213:889-94.

11. Harper PA, Kelly-Fry E, Noe JS, Bies JR, Jackson VP. Ultrasound in the evaluation of solid breast masses. Radiology. 1983; 146:731-6.

12. Hong AS, Rosen EL, Soo MS, Baker JA. BI-RADS for sonography: positive and negative predictive values of sonographic features. Am J Roentgenol. 2005; 184 : 1260-5.

13. Lee W-J, Chu J-S, Huang C-S, Chang M-F, Chang K-J, Chen K-M. Breast cancer vascularity: color Doppler sonography and histopathology study. Breast Cancer Res Treat. 1996; 37:291-8.

14. Jackson VP, Kelly-Fry E, Rothschild PA, Hoiden RW, Clark SA. Automated breast sonography using a 7.5 MHz PVDF transducer: preliminary clinical evaluation. Radiology. 1986; 159:679-84.
15. Lambie RW, Hodgden K, Herman EM, Kipperman M. Sonomammographic manifestations of mammographically detectable breast microcalcifications. J Ultrasound Med. 1983; 2:509-14.

16. Kasumi F. Can microcalcifications located within breast carcinomas be detected by ultrasound imaging?. Ultrasound Med Biol. 1988; 14(Suppl 1):175-82.

17. Guyer PB, Dewbury KC, Warwick D, Smallwood J, Taylor I. Direct contact B-scan ultrasound in the diagnosis of solid breast masses. Clin Radiol. 1986; 37 : 451-8.

18. Fornage BD, Lorigan JG, Andry E. Fibroadenoma of the breast: sonographic appearance. Radiology. 1989; 172:671-5.

19. Teubner J. The echogenic border: an important diagnostic criterion in sonographic tumor diagnosis of the breast. In: Gill RW, Dadd MJ, editors. World Fed Ultrasound Med Biol. Oxford:Pergamon; 1985. p. 342.

20. Bamber JC, De Gonzalez L, Cosgrove DO, Simmons P, Davey J, Mckinna JA. Quantitative evaluation of realtime ultrasound features of the breast. Ultrasound Med Biol. 1988; 14:81-7.

21. Kasumi F, Fukami A, Kuno K, Kajitani T. Characteristic echographic features of circumscribed cancer. Ultrasound Med Biol. 1982; 8:369-77.

22. Cole-Beuglet C, Soriano RZ, Kurtz AB, Goldberg BB. Ultrasound analysis of 104 primary breast carcinomas classified according to histopathologic type. Radiology. 1983; 147:191-6.

23. Venta LA, Dudiak CM, Salomon CG, Flisak ME. Sonographic evaluation of the breast. Radio Graphics. 1994; 14:29-50.

24. Ueno E, Tohno E, Itoh K. Classification and diagnostic criteria in breast echography. Jpn J Med Ultrasonics. 1986; 13:19-31.

25. Baum G. Ultrasound mammography. Radiology. 1977; 122:199-205.

26. Meyer JE, Amin E, Lindfors KK, Lipman JC, Stomper PC, Genest D. Medullary carcinoma of the breast: mammographic and US appearance. Radiology. 1989; 170:79-82. 